
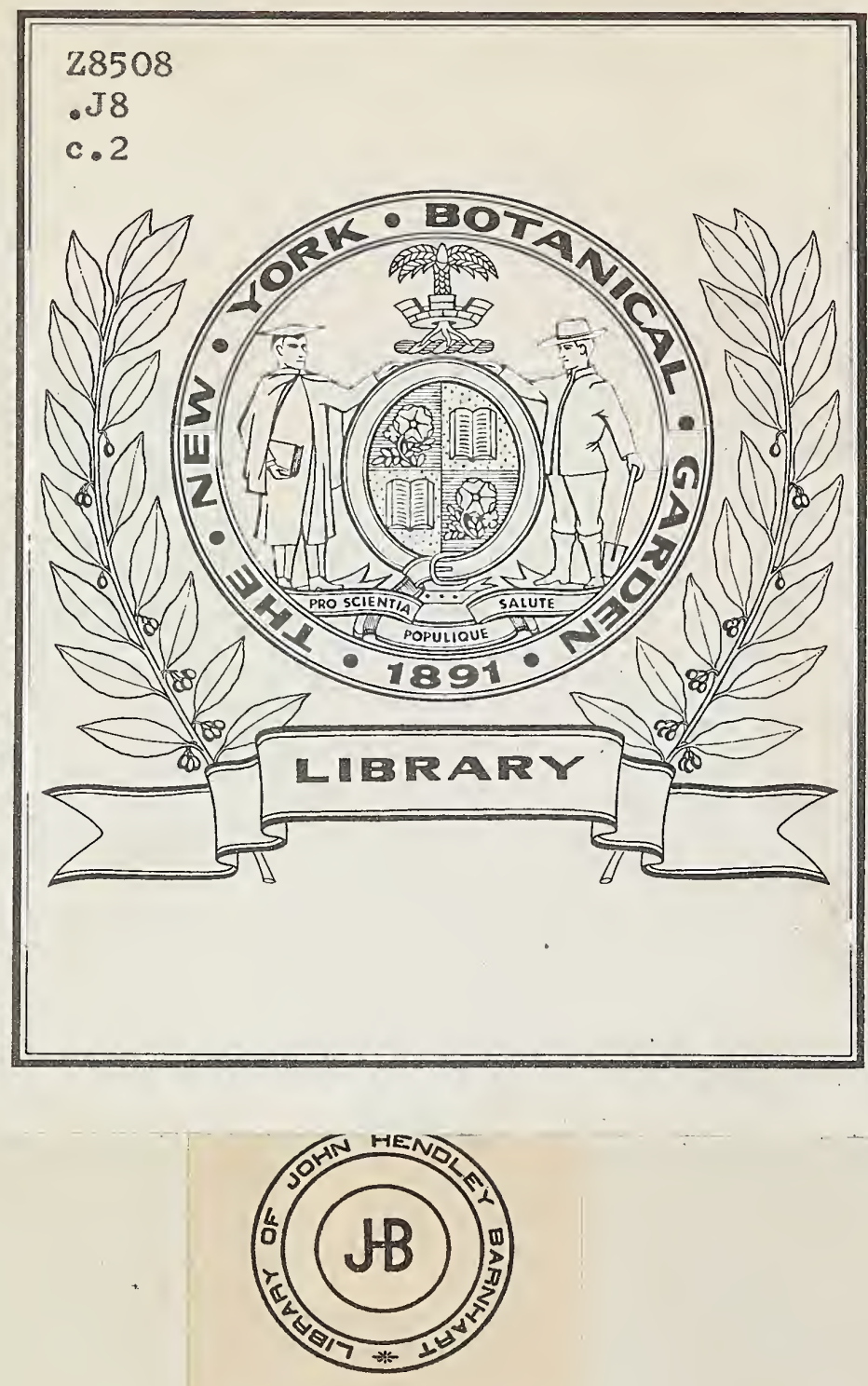



\section{Wilhelm Junk}

\section{Bibliographia Linnaeana.}

Verzeichniss der Schriften Karl von Linnés.

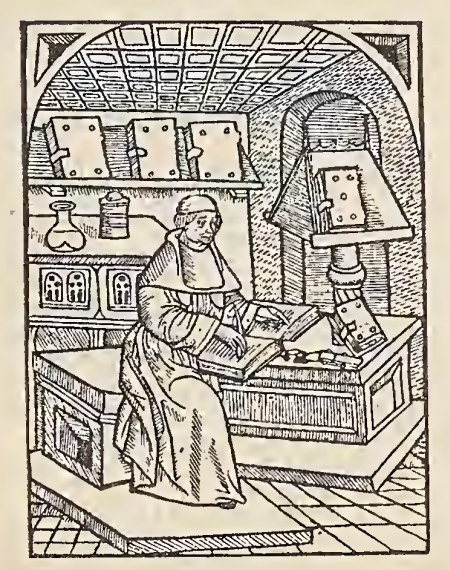

W. Junk

Berlin 1902.

Rathenower Str. 22. 


$$
\begin{aligned}
& z 8508 \\
& .28 \\
& c .2
\end{aligned}
$$




\section{C. a Linné.}

\section{(* 13. Mai 1707, + 10. Januar 1778).}

Systema Naturae. (Editio I.) Lugduni Batav., apud Th. Haak, 1735. 14 paginae in folio-max. M. 90.-14 unbezeichnete Seiten, von denen eine leer ist. Das 2. Blatt, das manchen Exemplaren fehlt, ist später erschienen, hat unten: Lugduni Batav. ap. A. Sylvium 1736, und enthält ein Schema.

Ist von R. Friedländer \& Sohn auf chemischem Wege zusammen mit dem Blatt: Methodus juxta quam Physiologus etc. und mit dem Bervic'schen Portrait von Linné (von 1779) in einer kleinen Zahl von Exemplaren (zu M. 15) vervielfältigt worden. Jetzt ebenfalls vergriffen. - Auch A. L. F ée hat diese Editio princeps u. d. T.: "Editio prima perrara reimpressa est curis ill. Fée“, bei Levrault in Paris im J. 1830 in einer Oktav-Ausgabe auf VI u. 81. Seiten neu gedruckt. Preis M. 5.

Linné selbst sagt von dieser Ausgabe: Conspectus tantum operis et quasi mappa geographica. Brunet 1862: très rare, 23 à 39 fr.!

2. - Editio II., auctior. Stockholmiae, G. Kiesewetter, 1740. 80 paginae in Octavo. Mr. 15.Linné: In hac editione emendavi multa, addidi plurima in omni naturae regno, praesertim vero species quadrupedum et nomina Suecana animalium.

3. - Editio III. Latine et Germanice per J. J. Lange. Halae, Gebauer, 1740. (8 et) 70 paginae in Octavo.

Identisch mit ed. II; nur die deutschen Namen hinzugefügt.

M. 4. -

Editio IV., ab auctore emendata et aucta, per B. Jussieu. Accesserunt nomina Gallica.

Parisiis, David, 1744. 108 paginae in Octavo.

Identisch mit ed. II; nur die französischen Namen hinzugefügt.

5. - Editio V. Cura M. G. Agnethler. Halae, Kümmel, 1747. 88 paginae in Octavo. (M. 0,60).

Identisch mit ed. II; nur wie bei der III. deutsche Namen hinzugefügt.

- Editio VI. - emendata et aucta. Stockholmiae, G. Kiesewetter, 1748. 18 et 224 paginae in

Octavo, cum 8 tabulis et effigie (Linnaei).

Gänzlich umgearbeitet. - Siehe auch Nr. 132.

7. - Editio VII. Secundum VI. Holmiensem editionem. Lipsiae, Kiesewetter, 1748.18 et 224

paginae in Octavo, eum 8 tabulis et effigie (Linnaei). Identisch mit ed. VI; nur deutsche Namen hinzugefügt.

8. - Editio VIII. Vegetabilia per J. J. Haartman, Regnum lapideum per H. Moeller. Holmiae

1753. 136 paginae in Octavo cum 3 tabulis.

Abdruck von ed. VI; aber die ,Thiere' sind nicht erschienen. In schwedischer Sprache.

- Editio IX. Per J. F. Gronovium. Lugduni Batav., Haak, 1756. 226 paginae in Octavo, cum 8 tabulis.

Identisch mit ed. VI., bis auf einige Veränderungen in den "Vögeln" und Fischen". Gleicher Herausgeber und derselbe Erscheinungsort wie die der ed. I. "Mit französisch"en Namen.

10. E d itio X., reformata. 2 volumina. Holmiae, Impens. T. Salvii, 1758-1759. in Octavo. M. 45.-Vol. I: Animalia, 1758. p. 1-824. - II: Vegetabilia. 1759. pg. 825-1384. (Mineralogie nicht enthaltend).

Gänzlich verändert und ausserordentlich vermehrt; die erste Ausgabe in zwei Bänden, zugleich aber die erste, in welcher die von Linné aufgestellte, zuerst in der 1. Ausgabe der ,Species Plantarum" von 1753 (siehe Nr. 115) veröffentlichte binäre Nomenclatur für Tiere und Pflanzen (für die Mineralien wird dies in der nächsten vom Autor besorgten, der XII., nachgeholt) durchgeführt ist. Daher nicht nur; wie die früheren Ausgaben, von geschichtlichem sondern auch von hohem wissenschaftlichem Werte. Ans diesem Grunde wurde auch ihr zoologischer (I.) Band von der Deutschen Zoologischen Gesellschaft nachgedruckt:

11. Systema Naturale. Regnum Animale. Fd. X., 1758. Cura societatis Zoologicae germanicae iterum edita. Lipsiae, Engelmann, 1894. VI et 824 paginae in Octavo. (M. 10). M. 8. 1759 erschien ferner:

12. Animalium Specierum method. dispositio secund. ed. X. Lugd. Batav., Haak, 253 pg. in Octavo.

1899 erschien:

13. C. D. Sherborn. Index to the generic and trivial Names of Animals described in the

10. and 12. edition. Manchester. $116 \mathrm{pg}$. in Octavo. 3.50

14. - Editio XI. Ad editionem X. reformatam Holminensem (recusa cura J. J. Lange). 2 volumina. Halae Magdeb., Curt, 1760. in Octavo.

Vol. I: Animalia. pg. 1-824. - If: Vegetabilia. pg. 825-1380.

Identisch mit ed. X. - Linné: Furtim prodiit vitiosa.

Ein 1765 in Haag bei Staalmann begonnener Nachdruck in Folio blieb, wohl wegen des Erscheinens der ed. XII., unvollendet.

45. - Editio XII. reformata. 3 volumina. Holmiae, Salvius, 1766--1768. in Octavo. Cum 3 tabulis.

Vol. I: Animalia. 1766. 1327 et 36 pg. - II: Vegetabilia. 1767. 736 et 16 pg. - IIJ: Mineralia; Appendix animalium et vegetabilium. 1768. 236 et $20 \mathrm{pg}$. et 3 tabulae.

Wiederum ausserordentlich vermehrt und wie die $\mathrm{X}$. ein fast neues Buch. Die wertvollste von allen Ausgaben, auch weil es die letzte ist, die der Autor noch selbst bearbeitet hat. (Siehe auch Nr. 13).

1771 kam heraus:

16. Genera Animalium ex ed. XII. Edinburgi 1771. 82 pg. in Octavo. M. 4.

1772 erschien:

17. Ex ed. XII. in epitomen redactum a. J. Beckmann. 2 vol. Gottingae, Vandenhoeck \& R. 240 et $356 \mathrm{pg}$. in Octavo, cum tabula: M. 6. 
18. - Editio XIII, ad ed. XII. reformatam Holmiensem. 3 volumina (in 4 partibus). Vindobonae, Trattner, 1767-1770. in Octavo. Cum 3 tabulis.

M. $15 .-$

Vol. I: (2 partes): Animalia. 1767. 1327 et 36 pg. - II: Vegetabilia. 1770. 736 et 16 pg. III: Mineralia etc. (wie oben). 1770.236 et $20 \mathrm{pg}$. et 3 tabulae. Have I. Identisch mit ed. XII.

19. - (Ed. XIV.) Editio XIII. aucta, reformata, cur. J. F. Gmelin. 3 volumina (in 10 partibus). Lipsiae, Beer, 1788-1793. in Octavo. Cum 3 tabulis. (M. 51).

M. $15 .-$

Vol. I (6 partes et index): Animalia. 1788. 4120 pg. - II (2 partes): Vegetabilia. 1791.40 et 1661 pg. - III: Mineralia. 1793. 476 pg. cum 3 tab. - Erschien auch mit Titel: Lugd. Batav., Delamollière, 1789-96. Have . T

20. J. A. Donndorf, Zoologische Beiträge zur 13. Ausgabe. 3 Bde. Leipzig, Weidmann, 1792 -98. Oktav. (M. 38). M. 9. - Nur Vertebraten enthaltend; wichtig.

Nur editio 1.. 2., 6., 10. und 12. sind Original-Ausgaben. Alle anderen sind Nachdrucke, die mit grosser Geschwindigkeit den Originalausgaben folgten, und die mit Ausnahme der letzten (posthumen) Gmelin'schen Ausgabe (die daher geschätzt wird) gleichlautend mit ihren Vorbilder'n waren. -

Eines der F'undamentalwerke der Naturwissenschaft, für welche das Jahr 1758 gleichwie 1859 den Anfang neuer Epochen bedeuten. Während das zuerst hier von Linné aufgestellte systèm, das den Zeitgenossen als das Wichtigste der reformatorischen Thätigkeit des Forschers erschien, heute zum Teil überholt speziell in der Botanik wieder verlassen worden ist, wird das, Systema seine dauernde Bedeutung als Originalquelle für die Nomenclatur der organischen Naturwissenschaften stets behalten. Speziell dem Zoologen ist das Buch als das einzige grosse, tierwissenschaftliche, das Linné geschrieben, von besonderer Wichtigkeit; der Botaniker muss, wie oben bemerkt, auf die editio princeps der ,Species Plantarum' zurückgehen. -- Eine interessante und nicht häufige Erscheinung ist der Umstand, dass das Erstlingswerk (im 28. Lebensjahre geschrieben) eines durch 4 Decennien literarisch so thätigen Gelebrten sein bedeutendstes geblieben ist.

Buchbändlerisch bedeutet erst das Erscheinen der ed. X. einen ausserordentlichen Preissturz der ganzen vorlinné'ischen Litteratur, soweit eine solche nicht geschichtliche Bedeutung oder Wert vom Standpunkte des Bibliophilen hat oder gute Tafeln besitzt - Die Preise der verschiedenen Ausgaben des, Systema، selbst sind in langsamem aber beständigem Steigen, woran der Neudruck der ed. I. nichts, und selbst der der ed. X. nur wenig änderten. Sogar die Preise der in Deutschland, Frankreich und Holland gemachten oft fehlerhaften Nachdrucke können sich dieser Tendenz nicht entziehen. Eine in ihrer Art einzige und bibliographisch interessante Besonderheit des Werkes bilden die allerdings wohlmotivierten Preis-Unterschiede der zahlreichen Ausgaben.

Es erschienen (geringwertige) Uebersetzungen in das Deutsche nach der ed. XII.) von P. L. St. Müller (11 Bände. 1773-1800 mit 195 (!) colorierten Tafeln), und von P. Hoeslin (2 Bde. 1781-82 mit 40 color. Tfln.) in das Englis che (nach der ed. XIII.) von W. Turton (7 Bände, mit 11 color. Tfln. 1800-1806) und von Sibly (14 Bde, m vielen color. Tfln. 1802), in das Französische von Vanderstegen de Patte (4 Bände, 1793), in das Holländische (nach der ed. XII.) von M. Houttyn (3 Bde. m. 296 Tfln. 1761-85). Auch von einzelnen Klassen wie Säugetieren und Insekten erschienen Uebersetzungen, die heute fast Makulatur sind.

Neubearbeitungen des Bandes II des ,Systema Naturae', ed. XII, erschienen unter dem Titel: 21. Systema Vegetabilium. Ed. XIII. Per J. A. Murray. Goettingae, Dieterich, 1774. 850 paginae in Octavo.

Nachtrag hierzu: -22. Supplementum Plantarum Systematis Vegetabilium edit. XIII., Generum Plantarum ed. VI. et Specierum Plantarum ed. II. (Editum a C. Linnaeo filio). Brunsvigae, Waisenhaus, 1781. $467 \mathrm{pg}$. in Octaro. M. 4.

Die Ausgaben des ,Systema Vegetabil.“ beginnen (da der Wiener Nachdruck „ad ed. XII.“ nicht als 13. betrachtet wurde und die $G$ melin'sche Ausgabe erst später erschien) mit „ed. XIII. " zu zählen.

23: Ed. XIV. edita a J. A. Murray. Goettingae, Dieterich, 1784. 907 pg. in Octavo. M. 3. -24. Ed. XV. praecedente longe correctior, curante J. A. Murray. Parisiis, Didot, $1798.837 \mathrm{pg}$. in Octavo. M. 4. - 25. (Ed XVI). Ed. XV. Procurata a Ch. H. Persoon. Goettingae, Dieterich, 1797. $1042 \mathrm{pg}$. in Octaro. M. 3. - 26-(Ed. XVII). Ed nova, aucta et locupletata. Curantibus J. J. Roemer. J. A. et J. H. Schultes. 7 vol. (in 8 partibus) et Mantissae 3 vol. Stuttgardiae, Cotta, 1817-30. in Octavo. (M. 127). IM. 20. - 27. (Ed. XVIII), Ed. XVI., curante K. Sprengel. 4 vol. (in 5 partibus) et Supplementum, auctore A. Sprengel. Goettingae, Dieterich, $1825-28$. in Octavo. (M. 60). M. 10.

Die Pariser Ausgabe von 1798 und die Stuttgarter (diese übrigens die beste von allen, trotzdem sie durch den Tod ihrer Herausgeber unvollständig blieb) von 1817-30 werden also von den Herausgebern der 4 Goettinger Editionen bei.der Zählung ignoriert.

28. Deutsche Uebersetzung: Vollständ. Pflanzensystem nach der 13. latein. Ausgabe. 14 Teile (in 15 Bdn.) Nürnberg 1777-88. Oktav. Mit 102 Tafeln. (M. 90). M. 15. - 28a. Nach der 14. Ausg. v. Murray, übersetzt v. J. Iippert. 2 Bde. Wien, Krauss, 1786 . Oktav. M. 4. - 29. Im Auszuge, v. B Merrem. 2 Bde. Marburg, Krieger, 1811. Oktar. (M. 9.) M. 4. - 29a. 2. Auflage. 2 Bde. 1823. Oktav. (M. 10.50). M."4.

30. Englische Uebersetzung: System of Vegetables. 2 vols. Lichfield $1783.897 \mathrm{pg}$. in Octavo with 11 plates. M. 5.

31. Französische Uebersetzung: Système sexuel des Végétaux. Par N. Jolyclerc. Paris, Ronvaux, 1798. 789 pg. in Octavo. (Fr. 9.25). M. 3. - 32. Edition II. 2 vols. Paris, Bertrand, 1810. in Octavo (Fres. 12). M. 4. - 33. Système des Plantes. Par J. P. Mo uton-Fontenille. 5 vols. Lyon 1804. in Octavo. M. 5. - 34. Linné français. 5 vols. Montpellier, Seguin, 1809. M. 5.

Das "Systema Plantarum", die "Species" und die „Mantissae" (siehe Nr. 70 u, 115) in ein Werk redigirt enthält:

Systema Plantarum. Editio novissima, novis plantis.... adaucta, curante J. J. Reichard.

4 vol. Francofurti, Varrentrapp, 1779-80. in Octayo. 
Ein nützliches Unternehmen ist das unter seinem Untertitel: „Codex Botanicus Linnaeanus" bekannte Work:

46 C. Linnaei Systema, Genera, Species Plantarum uno volumine. Editio critica. Edid. H. E. Richter. (In 14 partibus). Lipsiae, Wigand, 1835. 1134 paginae in Quarto. - Index alphabeticus Generum Specierum et Synonymorum. Compos. W. L. Petermann. Lipsiae, Wigand, 1840. 206 paginae in Quarto. (M. 48).

M. 12 . -

Enthält den ganzen Linnéschen Text. - Die Behauptung Brunet's: „Ce recueil a fait tomber le prix des éditions précédentes" hat, wenn sie überhaupt je richtig war," heut keine Berechtigung mehr.

37. Fundamenta Botanica. (Editio I). Amstelodami, Schouten, 1736. 36 paginae in Octavo. M. 12.38. Ed. II. Stockholmiae, Kiesewetter, 1740. 87 pg. in Octavo. M. 4. - 39. Ed. III. Amstelodami, Schouten, 1741. $51 \mathrm{pg}$. in Octavo. M. 3. - 40. (Ed. IV). Imprim. cum: Alston, Index Plantarum. Edinburgi 1740. 22 pg. in Octavo. M. 3. - -4t. (Ed. V). Imprim. cum: Linnaei Systema Naturae (ed. IV.). Parisiis, David, 1744. $26 \mathrm{pg}$. in Octavo, - 42. (Ed. VI). Halae, Bierwirth, 1747. 78 pg. in Octavo. M. 3. - 43. (Ed. VII). Impr. cum: Alston, Tirccinium Botanicum. Edinburgi $1753.27 \mathrm{pg}$. in Octavo. M. 3. - 44. (Ed VIII). Impr. in: Li nna ei, Opera varia. (vide nr. 131) Lucae, typ. Juntini, 17õ8. 61 pg. $\rightarrow 45$ (Ed. IX). Impr. cum: Linna ei. Systema Plantarum, curante J. E. Gilibert. Coloniae 1786. 48 pg. - 46. (Ed. X). Matriti 1788. 97 pg. in Octavo. M. 4.

Nur ed. I-III sind Original-Ausgaben. Die I. ist selten. - Die ,Fundamenta enthalten in 365 kurzen Thesen das Ganze der Botanik. Auf ihnen basieren zum grossen Teile die ,Bibliotheca Botanica6. die ,Critica Botanica', die ,Classes Plantarum,, die ,Oratio de Telluris Incremento' (Siehe im Folgenden). Das von Gilib ort als Fundamenta Botanica' (Bd. V-VII des ,Systema Plantarum') 1786-87 herausgegebene dreibändige Werk (siehe No. 148) bildet eine Sammlung botanischer Schriften Linne's, welche die eigentlichen ,Fundamenta: (No. 37) nur unter vielem Anderen enthalten. Tediglich eine commentierte Neu-Ausgabe derselben bildet:

47. Philosophia Botanica, in qua explicantur Fundamenta Botanica, cum definitionibus partium, exemplis terminorum, observationibus rariorum. (Editio I). Stockholmiae, Kiesewetter, 1751. 362 paginae in Octavo cum 11 tabulis. aloo $1755 . \quad$ IM. 3.48. Ed. II. Viennae, Trattner, 1763 (auch 1770). 368 pg. in Octavo cum 11 tabulis. M. 2. - 49- (Ed. III). Ed. II., curante J. G. Gleditsch. Berolini 1780. 362 pg. in Octavo cum 11 tabulis. et effigie M. 2. $-50-$ Ed. IV. Imprim. cum: Linnaei, Systema Plantarum, curante J. E. Gilibert. (Siehe Nr. 148). Coloniae 1787. 362 pg. in Octavo cum 13 tabulis. - - (Ed, V). Ed. III. aucta cura $\mathrm{K}$ L. Willdenow. Berolini 1790. 364 pg. in Octavo cum 11 tabulis. M. 2. - 52: (Ed. VI) Ed. IV, studio C. Sprengel. Halaè, Kümmel, 1809.362 pg. cum 11 tabulis. (M. 7). M. 2. 5ึ: (Ed. VII). Tornaci Nervior., typ. Casterman-Dieu, 1824. 497 pg. cum 9 tabulis. M. 3.

Nur die 1. Ausgabe ist Original." (J. P. Cobres: Ein vielbedeutendes Werk). Es erschien eine deutsche Uebersetzung (1787), eine englische (1775), eine französische (1788), und zwei spanische (1778 und 1792). - Die Tafeln, grossenteils Blatt-Formen und -Stellungen abbildend. sind dieselben wie die aus der Einleitung des "Hortus Cliffortianus' (siehe No. 78).

54. Bibliotheca Botanica, recensens plus mille de plantis hucusque editos ....., cum explicatione Fundamentorum Botanicorum, Pars I. Amstelodami, Schouten, 1736. 153 et 29 paginan in Octavo.

55. (Ed. II). Ed. nova. Halae, Bierwirth, 1747. 144 paginae in Octavo. M. 2. - 56. (Ed. III). Ed. altera, priori longe auctior et emendatior. Amstelodami, Schouten, 1751. $234 \mathrm{pg}$. in Octavo. M. 3.

Eine kritische Bibliographie. Die Ausgaben von 1736 und 1751 sind Original.

57. Musa Cliffortiana. Lugduni Batav. 1736. 46 paginae in Quarto cum 2 tabulis.

M. 3.-

Beschreibung einer im Clifford'schen Garten blïhenden Musa paradisiaca, deren lebende Blume man damals in Europa erst dreimal gesehen hatte.

58. Genera Plantarum (Editio I). Lugduni Batav., Wishoff, 1737. 384 paginae in Octavo. M. 15.59. Fd. II. aucta et emendata. Lugd., Wishoff, 1742.572 pg. in Octavo. M. 4. - 60: (Ed. III). Ed. II., nominibus plantarum Gallicis locupletata. Parisiis, David, $1743.445 \mathrm{pg}$. in Octavo cum 2 tab. M. 3. -61. (Ed. IV.) Curavit C. K. Strumpff. Halae Magdeb., Kümmel, 1752. 473 pg. in Octavo. M. 3. - 62 . Ed. V., ab auctore reformata et aucta. Holmiae, Salvius, 1754. 554 pg. in Octavo. M. 4. - 63. Ed. VI., ab auctore reformata et aucta. Holmiae, Salvius, 1764.642 pg. in Octavo. M. 4. - 64. (Ed. VII). Editio nova. Viennae, Trattner, 1767. in Octavo. M. 3. - 65: (Ed. VIII.) Ed. novissima, curante J. J. Reichard. Francofurti, Varrentrapp, 1778. 600 pg. in Octavo. M. 3. - $66-$ (Ed. IX). Ed. VIII., post Reichardianam secunda, curante J. Ch. D. v. Schreber. 2 vol. 1789-91. 904 pg. in Octavo. M. 3. - G7 (Ed. X). Ed. VIII., praecedente longe auctior, curante Th. Haenke. 2 vol. Vindobonae, Wappler, 1791.832 pg. in Octavo. M. 3. - 68. (Eb. XI). Ed. nova, curante C. Sprengel. 2 vol. Goettingae, Dieterich, $1830-31.876$ pg. in Octavo. (M. 12). M. 3.

Nur ed. I, II, V, VI sind Original-Ausgaben. - 69. Eine deutsche Uebersetzung von J. Plan er 2 Bde. m. Nachtrag. 1775-85. 1104 pg. M. 3.

Die ,Genera' beanspruchen als erste Veröffentlichung des Linné'schen, auf die Befiuchtungsorgane basierten Pflanzensystems geschichtliches Interesse. Die 1. Auflage enthält 9:35, die 6. bereits 1239 Arten. Als Supplement zu den, Genera', sowie auch zu den, Species (siehe No. 116) erschienen, die inzwischen gemachten Neu-Entdeckungen umfassend, und die Zahl der Arten auf 1335 bringend:

70. Mantissa Plantarum Generum editionis VI et Specierum editionis II. Holmiae, Salvius, 1767. in Octavo. Index et paginae $1-143$. - Mantissa Plantarum altera Generum ed. VI et

Specierum ed. II. Holmiae, Salvius, 1771. in Octavo. Index et paginae 144-588. . M. 40.Die 2. Mantissa entbält auch: Regni Animalis Appendix.

Von ausserordentlicher Wichtigkeit für die botanische Nomenclatur als Nachtrag zu dem. Fundamentalwerk „Species Plantarum". - Sehr'selten.

72. Corollarium Generum Plantarum. Accedit Methodus Sexualis. Lugduni Batav., Wishoff, 1737.

48 paginae in Octavo cum tabula.

M. 3.-

Erschien zusammen mit der ed. I der ,Genera' und ist lediglich ein Nachtrag zu derselben; 60 neue Genera (die 1742 in die ed. II mit einbezogen wurden) enthaltend. 
73. Viridarium Cliffortianum. Amstelodami 1737. 104 paginae in Octavo. Verzeichnis der Pflanzen, welche 1735-1737 in dem G. Clifford'schen Garten in Hartekamp blühten.

74. Flora Lapponica. Amstelodami, Schouten, 1737. 420 paginae in Octavo cum 12. tabulis. M. 7.75. (Ed. II) Editio altera, aucta et emendata, cura J. E. Smith. Londini, White, 1772.454 pg.

in Octavo, cum 12 tabulis. M. 6.

Nur die ed. I. ist Original. 100 neue Arten. Sehr geschätztes Werk, wichtig wegen der darin zuerst angewandten Methode. Pritzel schreibt: Prodromus immortalis operis 'Florula Lapponica' exstat in Act. liter. et scient. Sueciae 1732. p. 46-58; 1735. p. 12-23.

76. Critica Botanica... Seu Eundamentorum botanicorum, Pars IV. Accedit: J. Browallii de necessitate historiae naturalis discursus. Lugduni Batav., Wishoff, 1737. 294 paginae in Octavo. M. 3.7\%. (Ed. II). Imprim. cum: Linna ei, Systema Plantarum, curante J. E. Gilibert. (Siehe Nr. 148)

Coloniae 1787. pg. XXXII u. $363-594$.

Im wesentlichen ein ausgedehnter Commentar zu den Thesen 210--324 der Fundamenta, und später durch die ,Philosophia" ersetzt.

78. Hortus Cliffortianus. Amstelaedami 1737. 532 paginae in Folio cum 37 tabulis.

M. 25.Das äuserlich schönsteWerk Linné's, auf Kosten Clifford 's hergestellt, die'Tafeln von Eh ret gezeichnet. Neue Genera und Species. Von circa 2500 Pflanzen werden bereits die Synonyme gegeben. Sehr selten. nur in kleiner Auflage für Geschenke gedruckt. Die Seiten 232-300 existieren nicht. Brunet $30^{\prime}$ à $47 \mathrm{fr}$.

79: Classes Plantarum. Fundamentorum Botanicorum Pars II. Lugduni Batav., Wishoff, 1738.656 paginae in Octavo.

(Ed. II). Halae, Kümmel, 1747. 656 pg. in Octavo. M. 3.

Nur die 1738-Ausgabe ist Original. Ein eingehender Commentar der Thesen 53-78 der ,Fundamenta:

Im Jahre 1738 gab Linné seines eben verstorbenen Freundes Artedi's Lebenswerk heraus:

81. P. Artedi. Ichthyologia. Ed. C. Linnaeus: 5 partes. Lugduni Batav., Wishoff, 1738. in Octavo. Cum 4 tabulis.

M. 8.-

82. Oratio de Insectorum Proprietatibus seu Memorabilibus. Holmiae 1739. 20 paginae in Octavo. M. 3.-Abgedruckt in den ,Amoenitates Acad.' (siehe No. 105). - 2 deutsche Uebersetzungen (1770), eine holländische (1741), eine schwedische (in 3 Auflagen, von denen die beiden letzten identisch, 1739-1752). - Die erste öffentliche Rede Linné's.

83. Oratio qua Peregrinationum intra Patriam asseritur necessitas. Upsaliae 1741 . 48 paginae in Octavo.

83a. (Ed. II): Oratio de Necessitate Peregrinationum intra Patriam. (pg. 1-28.) Ejusque: Elernchus Animalium per Sueciam observatorum (pg. 37-94). Lugd. Bat, Haak, 1743. in Octevo. M. 4. Auch in den ,Amoenitates Acad.' alogedruckt, ebenso in Linnaei Systema Plantarum, curante J. E. Gilibert, 1786 .

Antritts-Vorlesung. Die 2. Rede Linné's.

Orbis eruditi / Judicium | De | Caroli Linnaei | M. D. | Seriptis. (Holmiae 1741). 16 paginae in

Octavo.

85. (Ed. II). Facsimile-Edition. Ed. W. Junk. Berolini 1901. 16 pg. in Octavo. M. 10.

M. $120 .-$

Stöver, Leben Linné's I. (pg. 240-257):

Dies ist die einzige besondere Apologie, die Linné jemals für sich geschrieben (nach der akademischen Schrift: Decades binae thesium medicarum, die sein erbitterter Gegner, der Mineralos Johann Gottschalk Wallerius, am 25. Februar 1741 öffentlich zu Upsala verteidigt hatte), sowie die einzige Schrift, die er anonymisch herausgegeben hat. Diese Schrift ist äusserst selten, selbst in Schweden. Weder Haller noch andere Literatoren scheinen sie gekannt zu haben, haben sie wenigstens nicht angeführt, und in Teutschland existiert sie vielleicht nicht in ein paas Exemplaren. Die Merkwürdigkeit, sowohl als die Unbekanntheit des Inhalts derselben verdienen daher eine nähere Darstellung. Der Titel enthält den Wablspruch Linné's aus dem Virgil: Famam extoller'e factis-hoc virtutis opus; und auf der Rückseite die Inschrift Gronow's auf Linné: Ne succumbe malis; Te noverit ultimus Ister, Te Boreas gelidus. Alsdann folgt eine kurze Uebersicht der vorzüglichsten Lebensmerkwürdigkeiten Linné's und ein Verzeichnis seiner bis dahin erschienenen Schriften, mit ibren verschiedenen Ausgaben - zusammen 21, nebst einer Angabe der Männer, die das Linné'sche System öffentlich angenommen und verteidigt haben: van Royen, Gronow, Ferber, Browallius, Gleditsch. Hierauf werden die gedruckten oder in Briefen geäusserten Urteile und Zeugnisse von zwanzig Gelehrten über Linné mitgeteilt: Johann van Gorter, Boerhaave (,Saecula laudabunt, boni imitabuntur, omuibus proderit"c), van Royen, Gronow, Burman; Hans Sloane, Dillenius, Lawson, Donell, Jacob; De Saurages, Antoine de Jussieu, Barrère, Gravel; Albrecht von Haller, Johann Gesner, Gleditsch, Breyne. Johann Joachim Lange, Friedrich Otto Menken, F. Thomas Kohl." - Vergleiche auch: Desgenettes, Notice sur un opuscule rare relatif à Linné (Journal complémentaire des Sciences médicales. Tome XXX. 1838). - Pritzel, der das Werkchen in der 1. Auflage nicht citiert, beginnt, nachdem er ein einziges Exemplar in der Bibliothek von $D_{e}$ Candoll e gesehen hat, in der 2. Auflag'e die ausführliche Beschreibung desselben mit den Worten: De hoc rarissimo libello.

Ausser dem obigen im Besitz von W. Junk befindlichen sind nur noch drei andere bekannt, von denen zwei am 18. November 1888 in der Auction A ehrling in Stockholm vorkamen. Gegenüber diesem Rarissimum verschwinden in Bezug auf Seltenheit die vielen anderen gesuchten Schriften Linné's, selbst die editio princeps des 'Systema Naturae'. Es ist eine der seltensten Flugschriften ïberhaupt.

Auch der neue Nachdruck, der die Kenntnis des Werkchens vermittelt. ist auf das günstigste aufgenommen worden. (Siehe u. a. Prof. Nitsche-Tharand im, Literar. Centralblatt', Prof. WittBerlin im ,Prometheus', F. v. Zobeltitz-Berlin in der ,Zeitschrift für Bücherfreunde'.)

86. Oratio de Telluris habitabilis Incremento. Lugduni Batav. 1744. in Octavo.

3. Rede. Auch Discussion der 132. These der ,Fundamenta: 
-87. Oeländska och Gothländska Resa . . förrättad åhr 1741. Stockholm, Kiesewetter 1745.389 paginae in Octaro. Selten. Botanisch wichtig.

88. Ed. II: Gothländska Resa. Nya Uplag. Stockholm 1890. 116 pg., Oktav, mit 1 Karte. M. 2. 89. Deutsche Uebersetzung von J. C. D. Schreber erschien 1764 zu Halle: 416 Seiten in Oktar mit 4 Tafeln u. Karten. M. 3. (Auch u. d. Titel: Reisen durch einige Schwedische Provinzen, Teil 1).

Flora Suecica. Stockholmiae, Salvius, 1745. 431 paginae in Octavo cum 1 tabula. M. 4.91. Ed. II, aucta et emendata. Stockholmiae, Salvius, 1755. 494 pg. in Octavo cum 1 tab. M. j.

Die 1. Auflage umfasst 1140, die zweite 1296 Pflanzen. Die 1. Ausgabe scheint auch mit der Jahreszahl $170 \tilde{1}$ erschienen zu sein.

92. Fauna Suecica. Stockholmiae, Salvius, 1746. 411 paginae in Octavo cum 3 tabulis. M. 3.-Die Ausgabe mit Titelblatt: Lugd. Batav., Wishoff, 1746. ist identisch. - 93. Ed. II. auctior. Stockholmiae, Salvius, 1761. 604 paginae in Octavo cum 3 tabulis. M. 6. - 94. (Ed. III). Emend. A. J. Retzius. Tomus I (quantum prodiit): Vertebrata. Lipsiae, Vogel, 1800. in Octavo. Cum tabula color. M. 4.

Erste Ausgabe 1350, die zweite, an welcher L,'s Schüler mitarbeiteten, 2266 Arten. Hauptsächlich entomologisch.

95. Flora Zeylanica, sistens plantas indicas Zeylonae insulae, quae olim 1670-77 lectae fuere a P. Hermanno. Holmiae, Salvius, 1747. 240 paginae in Octavo cum 4 tabulis. M. 4.96. (Ed. II). Amstaelodami, Wetsten, 1748. in Octavo. M. 4. (Ist identisch).

97. Wästgöta-Resa, . . förrättad år 1746. Stockholm, Salvius, 1747, 284 paginae in Octavo cum 5 tabulis.

Die seltenste seiner Reiseń. - -98. D e utsche Uebersetzung. von J. C. D. Schreber erschien $1765 \mathrm{zu}$ Halle: 333 Seiten in Oktav mit 7 Tafeln. M. 4. (Auch u. d. Titel: Reisen durch einige Schwedische Provinzen; Teil 2).

9. Hortus Upsaliensis, exhibens plantas exoticas horto Upsaliensi . . illatas ab anno 1742 in annum 1748. Volumen I (quantum prodiit) .Stockholmiae, Salvius, 1748. 306 paginae in Octavo cum 3 tabulis.

100. Materia Medica. Liber I (quantum prodiit) de Plantis. Holmiae, Salvius, 1749. 252 paginae in Octavo cum 1 tabula.

101. (Eid. II). Ed. altera auctior. Cur. J. C. D. Schreber, Lipsiae 1772. in Octavo. Cum tabula. M. 2. (Auch zoologisch und mineralogisch). - 102. (Ed. III). Curante Schreber. Vindobonae 1773. in Octavo. M. 2. - 103. Ed. IV., auctior, curante Schreber. Lipsiae 1782. in Octavo. M. 2. - 104. Ed. V., auctior, curante Schreber. Lipsiae, Breuning, 1787. 318 pg. in Octavo. M. 3.

105. Amoenitates Academicae, seu dissertationes physicae, medicae, botanicae. 7 volumina. Holmiae, Kiesewetter et Salvius, 1749-1769, in Octavo. Cum 44 tabulis. (M. 42). M. 30.Tomus 1 : 1749. $563 \mathrm{pg}$. et 17 tab. - 任. $1752.478 \mathrm{pg}$. et 4 tab. (Ed. Il. aucta. 1762. 444 pg. et 4 tab.) - 1 te. 1756.464 pg. et 4 tab. - IV. 1760. 600 pg. et 4 tab. - F. 1760.483 pg. et 3 tab. - VT. 1763. 486 pg. et 5 tab. - VII. 1769. 506 pg. et 7 tab.

106. (Ed. II). 7 vol. Lugd. Batav, et Amstelod. 1749-1769. in Octavo. Cum 42 tab. M. 30. (Die Bände dieses holländischen Nachdrucks erschienen mit Ausnahme des 2., welcher 1764 herauskam, in denselben Jahren wie das Original). - 107. (Ed. III). Curante J. Ch. D. Schreber. 10 vol. (Auf: den Titelblättern von Bd. 1-3: ed. III.; 4-7: ed. II. ; 8-10 isted. I.). Erlangae, Palm, 1787-1790. in Octavo. Cum 59 tab. (Bd. 10: C. a Linné filius: Dissertationes botanicae collectae). (M. 54). M. 20.

Auszug: 108. Selectae ex Amoenit. acad. Dissertationes. (Ed. L. Biwald). 3 vol. Graecii, typ. Wid- har manstadt, 1764-67. in Quarto. Cum 7 tab. M. 5. - 109. Ed. II. 1 vol. Graecii, Zaunrieth, 1786. $285 \mathrm{pg}$. in Octavo. Cum 3 tab. M. 3. - 110. Deutsche Uebersetzung dieses Auszuges von Th. Ho ep fner. Leipzig, Böhme, 1776-78. Octav. Mit 15 Tafeln. M. 3. - Auch von Gilibext scheint unter dem Titel: 11. Amoenitates selectae $1785 \mathrm{zu}$ Lyon in 2 Bänden ein Auszug erschienen zu sein. - Ein englischer Auszug erschien unter dem Titel: 112. Miscellaneous Tracts, by B. Stillingfleet. London 1759. $230 \mathrm{pg}$. in Octavo w. 11 plates. M. 4; 2. edition. London, Dodsley, $1762.423 \mathrm{pg}$. in Octaro with 11 plates. M. 5. Supplement by F. J. Brand. Vol. I. (all published). London $1781.480 \mathrm{pg}$. in Octaro. M. 3.

Unter diesem eigentümlichen, aber um jene Zeit beliebten Titel, speziell gebraucht für Werke, die nicht aus einem Guss bestanden, sondern mehr systemlose Aneinanderreihungen von Arbeiten und Beobachtungen eines oder mehrerer Autoren bildeten (siehe: Roesel's Insektenbelustigung, Köhler's Münzbelustigungen, Knor's's Deliciae Naturae, Ledermüller's Amusemens microscopiques, und viele andere) - ist hier eine Sammlung von 186 kleinen medizinischen und naturwissenschaftlichen (unter letzteren meist - 93 - botanische, auch 25 entomologische) Abhandlungen vereinigt. Alle diese, vorher auch schon einzeln erschienen. sind Dissertationen, die unter dem Rectorate Linné's geschrieben und unter den verschiedensten Autor-Namen veröffentlicht wurden, jedoch sind sämtliche von $\mathrm{L}$. beeinflusst, ja zum überwiegenden Teil sogar von ihm verfasst. (Ein genaues Verzeichnis und Analyse giebt Pulteney, Nr. 186). Sie enthalten neben vielem Wertlosen (z. B. den medizinischen Abhandlungen) manche wichtige Vorarbeit zu später gedruckten grössèren Werken. Viele der Abhandlungen sind später auch einzeln in Schwedischer, einige in Deutscher und Englischer Uebersetzung erschienen.

Komplette Exemplare, besonders solche der Stockholmer Original-Ausgabe, welche durch Band 8-10 der Erlanger vervollständigt sind, werden selten und steigen im Preise. (Bru n et 1862: 40 à 50 fr.). Unvollständige Reihen und einzelne Abhandlungen in Original oder Uebersetzungen besitzen nur geringen Wert.

113. Skånska Resa . . . förrättad år 1749. Stockholm, Salvius, 1751. 448 paginae in Octavo cum mappa geograph. et 6 tabulis.

Selten.

414. Ed. II. Lund 1874. M. 4. - 115. Deutsche Uebersetzung (v. K. E. Klein): Reisen durch das Königr. Schweden. Leipzig, Kiesewetter, 1756. 336 pg. in Oktav mit 1 geograph. Karte und 2 Tfn. M. 6. 
115. Species Plantarum. (Ed. I). 2 vol. Holmiae, Salvius, 1753. 1200 paginae in Octavo. M. 55.116. Ed. II., aucta. 2 vol. Holmiae, Salvius, 1762-63. 1862 pg. in Octavo. M. 25.117. Ed. III. 2 vol. Vindobonae, Trattner, 1764. 1862 pg. in Octavo. M. 6.118: Ed. IV., post Reichhardianum ed. V. (siehe No. 35). Curante C. L. Willdenow, contin. a F. Schwaegrichen et H. F. Link. 6 vol. (in 12 partibus). Berolini, Nauck, 1797-1830. (M. 76). M. 20. - (Hierzu erschien 1803, und in 2. Auflage 1821, ein 119., Nomenclator Botanicus' von F. Henckel zu Donnersmarck. - Siehe anch No. 145). - Eo. Ed. VI. Auctore A. Dietrich. Tomus I et II (quantum prodiit). Berolini, Nauck, 1831-33. 745 et 747 pg. in Octavo. (M. 18). M. 4.

Supplemente - siehe No. 70: Mantissa Plantarum.

121. J. A. Schultes, Observationes botanicae in Linnaei Species Plantarum ex editione Willdenow. Oeniponti, Wagner, $1809.232 \mathrm{pg}$. in Octavo. M. 2.

122. F. d e Mueller, Index ad Linnaei Species Plantarum ed. I. Melbourne 1880. in Octavo. M. 2.50. Nur ed. I. und II. sind Originalausgaben, die IIl. ist ein wortgetrener Abdruck. Von ed. IV. ab überwiegt in den Ausgaben die Arbeit der Herausgeber.

Die ,Species Plantarum" sind nach dem ,Systema Naturae" das hervorragendste Werk Linné's, welches seinen Ruhm für immer bewahren wird; ja sie sind in gewisser Hinsicht, speziell für die Botanik, ebenso wichtig, weil, wie bemerlkt, die binäre Nomenclatur, L.'s bleibendes Verdienst, hier zum ersten Male eingeführt wurde. Sie fanden merkwürdigerweise keinen Uebersetzer. Die Preise der einzig wichtigen beiden Erst-Auflagen sind seit 1) Jahren um das dreifache gestiegen und werden, sollte nicht wie bei dem zoologischen Teil der ed. X. des ,Systema' (welcher für die Zoologie genau dieselbe Rolle spielt wie die "Species' für die Botanik) ein Neudruck erfolgen, sicher noch viel mehr erhöht werden. Brunet 1862: éd. I. 10 à 12 fr.

123. Museum Tessinianum. Holmiae, Salvius, 1753. in Folio. Cum 12 tabulis (M. 12).

M. $7 .-$

Beschreibung des Mineralien- und Versteinerungs-Cabinets des Grafen C. G. Tessin. Lateinisch und Schwedisch.

124. Museum Adolphi Friederici Regis Suecorum. Holmiae, Momma, 1754. 133 paginae in Folio cum

33 tabulis. (M. 72). $\quad$ M. 20.-

In Band I der ,Amoenitates" war 1746 bereits ein Vorlëufer, 48 Seiten mit 2 Tafeln (,anctore L. Balk"), erschienen.

Beschreibung seltener Tiere des kgl. Cabinets. Wichtig wegen der Abbildung von 48 Fischen und 32 Schlangen, auf welche L. im ,Systema' sich häufig bezieht. - Selten. Brunet: 25 à 50 fr., rare.

125. Prodromus Musei Adolphi Friederici Regis. Holmiae 1765. 110 paginae in Octavo. M. 5.-

Nachtrag :zum obigen, 156 seltenere Vertebraten enthaltend. Wohl der Vorläufer eines - niemals erschienenen -. Bandes II des 'Museum'.

In Jahre 1757 gab Linné nach dem Tode des Autors heraus:

126. F. Hasselquist, Iter Palaestinum . . förrättad ifrån år 1749 til 1752 utgifvn. af C. Linnaeus. Stockholm, Salvius, 1757. 619 pg. in Octavo.

127. Deutsche Uebersotzung v. Th. H. Gadebusch. Rostock, Koppe, 1762. 606 pg. in Oktav. M. 4. 128. Englische Uebersetzung. London 1766. in Octavo. M. 4. - 129. Französische Uebersetzung v. Eidous. 2 Teile. Paris, Saugrain, 1769. in Octavo. M. 4.

Faunistisch und floristisch:

Im folgenden Jahre veröffentlichte er ebenfalls nach dem Tode des Autors, seines Schülers:

130. P. Loefling, Iter Hispanicum . . . förrättad ifrån år 1751 til 1756. Utgifven af C. Li nnaeus. Stockholm, Salvius, $1758.316 \mathrm{pg}$. in Octavo cum 2 tabulis. M. $5 .-$ 130a. D eutsche Tebersetzung v. A. B. Ko el pin. Berlin, Lange, 1766. 406 pg. in ()ktav mit 2 Tafeln. M. 4. (Die Ausgabe mit Jahreszahl 1776 ist wohl identisch). - 132. Englis che Uebersetzung. London 1771. in Octavo.

Faunistisch und floristisch.

131. Opera varia, in quibus continentur Fundamenta Botanica, Sponsalia Plantarum et Systema Naturae. Lucae, typ. Iuntini, 1758. 376 paginae in Octavo cum tabula. M. 4.132. Deutsche Uebersetzung: Auserlesene Abhandlungen. 3 Teile. Leipzig, Böhme, 1776_78. Oktav. Mit Tafeln. M 3.

Eine von Linné nicht autorisierte Sammlung. Auf Seite 147-376 Abdruck der ed. VंI. des ,Systema:

133. Disquisitio de quaestione . . . : Sexum Plantarum argumentis et experimentis novis vel corrobare vel impugnare. Petropoli, Academia Sc., 1760. 30 paginae in Quarto.

134. Englische Uebersetzung: Dissertation on the Sexes of Plants. By J. E. Smith. London, Nicol, $1786.77 \mathrm{pg}$. in Octavo. M. 6 .

Von der Petersburger Akademie gekrönte Preis-Schrift. - Selten.

135. Termini Botanici. Upsaliae 1762. 32 paginae in Quarto.

M. 2.-

Erschien, so wie oben beschrieben, zuerst in dem VI. Band der ,Amoenitates Academicae und dürfte wohl die einzige Abhandlung aus diesen sein, die später in unübersetzter Sonderausgabe erschienen ist.

136. (Ed. II). Editio ancta a Schreber. Lipsiae, Crusius, 1767 (auch 1768). in Octavo. M.2. - 137. (Ed. III). Aucta a J. Rotheram. Novi Castri 1779. in Octavo. M. 2. - 138. (Ed. IV). Cur. P. D. Gi s eke. Hamburgi, Hoffmann, 1781 (und 1787). 219 pg. in Octavo. M. 2. - 139. (Ed. V). Editio IV. aucta. Hamburgi, Herold, 1789.396 pg. in Octavo. M. 2. - 140. (Ed. VI) Erlangae, Palm, 1789 (und 1792). in Octaro. M. 2. - Ausserdem wurde die Abhandlung mitabgedruckt in Systema Plantarum, ed. Gilibert, 1785, und in Hudson's Flora Anglica, ed. II. 1778. - 141. Schwedische Uebersetzung v. B. A. Euph rasén. Gotheborg, Wahlström, 1792. 74 pg. in Octavo. M. 2.

142. Museum Ludovicae Ulricae Reginae. Holmiae, Salvius, 1764. 728 paginae in Octavo. M. 12.Blos entomologisch und conchyliologisch. Wichtig, weil die ed. XII. des Systema ofters auf dasselbe zurückgreift. - Selten. Brun et: Peu commun et fort recherché, 23 à $44 \mathrm{fr}$. 
14. Clavis Medicinae. Holmiae 1766. 29 paginae in Octavo.

144. Ed. II. Cum praefatione Bal dinge r. Langensalza, Mariini, 1767. in Octavo. M. 2.

145. Nomenclator Botanicus, enumerans Plantas omnes in Systematis Naturae edit. XII., Specier. Ist aber nicht von linné herausgegeben. - Siehe auch No. 119.

146. Deliciae Naturae. Stockholm, Lange, 1773. 39 paginae in Octavo.

M. 3.-

147. Ed. II. Stockholm, Sohm, 1816. 42 pg. in Octavo. M. 3.

In Schwedischer Sprache. - In Lateinischer später in den, Amoenitates' abgedruckt. Der von Stoever erwähnte lateinische Einzel-Druck dieser bei Niederlegung des Rektorats gehaltenen Rede ist nach Wikstroem niemals erschienen.

148- Systema Plantarum Europae. Curante J. E. Gilibert. 7 vol. Coloniae, Piestre, 1785-87. in Octavo. Cum 13 tabulis. $\quad$ M. 20.Bd. V-VII, 1786-1787, haben den Titel: Fundamenta Botanica Linnaei (Siehe Nr. 37). - Enthält eine Sammlung der botanischen Fundamentalwerke L.'s, aber auch - (und diese sind wichtiger) - Werke von Gilib ert: die ,Flora Lithuanica' und die ,Chloris Lugdunensis'; ferner die ;Flora Delphinalis' von D. Villar. - 149. Gilibert, Exercitia phytologica. 2 vol. Lugd. Gallorum, Delamollière, 1792. 735 .pg. c. 106 tabulis. (Inh.: Plantae Lithuanicae). M. 45. (Sehr selten). Bildet ein Supplement zu obigem Werke.

150. Entomologia faunae Suecicae descriptionibus aucta ... Curante C. de Villers. 4 vol. Iugd., Piestre et Delamollière, 1789. in Octavo. Cum 11 tabulis. (M. 45). Hat mit Linné's Werk weniger zu thun, da auch die Bücher von Scopoli, Geoffroy, De Geer, Fabricius, Schrank u. a. mit hinein verarbeitet worden sind.

151. Praelectiones in Ordines naturales Plantarum. E proprio et J. Ch. Fabricii edidit P. D. Giseke. Hamburgi, Hoffmann, 1792. 712 paginae in Octavo. Cum 8 tabulis.

Lachesis Lapponica. Tour in Lappland, now first published by J. E. Smith. 2 vols. London 1811. in Octavo. Wichtig und selten.

153. Exercitatio botanico-physica de Nuptiis et Sexu Plantarum. Ed. J. A. Afzelius. Upsaliae, typ. Acad., 1828. 49 paginae in Octavo.

M. 3. Lateinisch u. deutsch. Ueber die merkwürdige Geschichte dieses Werkchens, siehe Pritzel, ed. II. 5435.

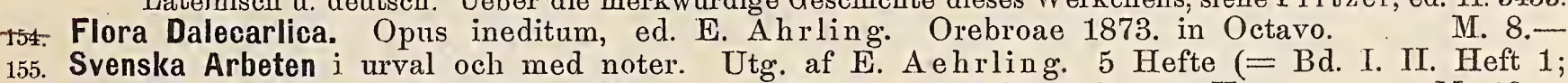
soviel erschienen). Stockholm 1878-80. Oktav. Mit 1 Portrait u. 1 Karte. M. 10.-

156. Ungdoms-Skrifter. Samlade af E. A ehrling, utgifna af Vetenskaps-Akademien. 2 Bände. Stockholm, Nordstedt, 1888--89. Oktav. Mit Karte u. Tafel.

Enthält u..a.: Iter Lapponicum. Iter Dalekarlicum. Iter ad Exteros.

157. Hortus Uplandicus. Med inledning och forklaringar af T. M. Fries. Upsala 1899. 48 paginae in Octavo cum 2 tabulis.

Ausserdem erschienen in den Jahren 1732-1770 verschiedene kleinere Abhandlungen (die übrigens wohl sämtlich in den "Amoenitates" wieder abgedruckt wurden) in den "Acta Societatis Regiae Scientiarum Upsaliensis".

Ich erwarb soeben ein in hohem Grade interessantes Unicum:

C. a Linné, Collegien-Hefte, nach dessen Vorlesungen geschrieben von Friedrich Mozelius. 4 Teile. Upsala 1748. 423 Klein-Quart-Seiten in sauberer Handschrift. In gleichzeitigem Papp-Band.

M. 30.-

Inhalt: Archiater Linnaei Föreläsningar öfver 'Fundamenta Botanica', häldne i Upsala 1748. 90 Seiten. - Clavis in Regnum Vegetabile. 1748. 33 Seiten. - Bemärkningar af Archiaterns Herr Caroli Linnaei enskilden föreläsningar uti Natur-Historien 1748. (Regnum Lapideum. -.. Rein Mineralogisch). 208 Seiten. - Anmärkningar under Herr Arch. Linnaeus uti Zoologien. 1748. 92 Seiten.

Diese in schwedischer Sprache von einem Studenten nachgeschriebenen Vorlesungen, die L. während eines oder zweier Semester hielt, dürften die einzigen Collegienhefte sein, die erhalten geblieben sind. Linné stand gerade danals, in seinem 41. Lebensjahre, auf der Höhe seines Schaffens. Durch einen glücklichen Umstand hat uns der Band die Vorlesungen über sämtliche drei Naturreiche überliefert. Der Schreiber, Student Mozel, der jedenfalls Arzt wurde, scheint sonst nichts für die Nachwelt geleistet zu haben.

Auch der sehr rege Briefwechsel L.'s wurde nach dessen Tode verschiedentlich gesammelt und publiciert. 158. Collectio Epistolarum qu. ad viros illustr. et claros scripsit. Ed. D. H. Stoever. Hamburg, Hoffmann, 1792. 208 paginae in Octavo. (M. 160). $\quad$ M. 2.50

159. Correspondence of Linnaeus and other naturalists from the original manuscripts, edited w. biograph. memoir by J. E. S mith. 2 volumes. London 1821. in Octavo. With facsimiles $(1 £ 10 \mathrm{sh}$.). M. 8.-

160. Epistolae ineditae 1736-93. Ed. H. C. van Hall. Groningen, Boekeren, 1830. in Octavo. (fl. 2.70).

161. Literae ineditae virorum celebrium Danorum ad Linnaeum. Ed. a J. C. Schiödte (Kjöbenhavn) 1870. 190 paginae in Octavo.

162. C. v. Linnés Brefväxling. Förteckning, upprättad af E. A e hrling. Stockholm 1885. Oktav. M. 2.50

Von Briefen, die L. mit einzelnen Gelehrten wechselte, kamen heraus:

163. Lettres inédites de Linné à Boissier de la Croix de Sauvages. Recueillies par d'Hombres Fir.mas. Veirun 1860. 302 pages in Octavo.

164. Gmelini Reliquiae quae supersunt commercii epistolici cum Linnaeo Hallero et a., ed. Plieninger. Stuttgart. in Octavo. Cum autograph. 
165. Epistolae ad N. J. Jacquin, ed C. N. J. v. Schreibers. Vindobonae, Gerold, 1841 . in Octavo. (M. 4).

166. Epistolae ad B. de Jussieu ineditae. Curante A. d e Jussieu. Cambridgiae Nov. Angl. 1854. in Quarto. 467. Lettres à David van Royen publiées et annotées par E. Bonnet (Genève, Bull. Herb. Boiss.) 1895. 14 pages in Octavo.

M. 1.50

168. Lettere inedite a G. A. Scopoli. Pubblicate da G. de Cobelli e C. Delaiti. Rovereto 1889. 26 pagine in Ottavo.

M. 2.-

Nicht gering ist erklärlicherweise die biographische Litteratur über L. Er selbst hinterliess:

169. Egenhändiga Anteckningar af Linnaeus om sig sjelf. Med anmärkn. och tilläg (af A. Afzelius). Stockholm 1823. 272 Seiten in Quart. Mit 6 Tafeln.

M. 7.-

170. Deutsche Uebersetzung v. Lappe. Berlin, Reimer, 1826. 280 Seiten in Oktav. Mit Portrait und 2 Tafeln. (M. 3.60).

M. 2.-

171. Lefnads-Minnen tecknade af honom sjelf. Med tillägg af A. A hnf elt. Stockholm 1877. Octav. M. 2.50

Von Biographien aus fremder Feder sind die wichtigsten:

172. C. A. Agardh, Antiquitates Linnaeanae.: Lund, Berling, 1826. 18 paginae in Folio. M. 4.Programm. - Auch schwedisch in: Svenska Akad. Handlingar. Bd. X 1826. (Seite 49-108). (Dieses 1863 noch einmal abgedruckt).

C. A. Agardh, Vie de Linné. Paris 1832. 390 pages in Octavo avec 6 planches. M. 6.-

174. A. Alberg, Linnaeus, the floral King. A Life of L. London 1888. 240 pages in Octavo. Cloth. M. 5. 175. (Miss) Brightwell, The Life of Linnaeus. London, Van Voorst, 1858. 191 pages in Octavo. (3 sh. 6 d.)

176. A. Cattaneo, Cenni su la vita di Linné. Milano 1838. in Quarto. Con ritratto. M. 3.-

M. 3.-

177. A. L. Fée, Vie de Linné, réd. s. les documens autogr. (Lille, Mém. Soc. Roy., 1831). in Octavo. Avec autogr.

A paru aussi séparément à Paris en 1832.

M. 8. -

T. M. Fries, Bidrag till en Lefnadsteckning. Teil 1-8 (soviel erschienen). Upsala 1893-99. 502 paginae in Octavo.

M. 10.

179. J. Gistel, Linnaeus. Ein Lebensbild. Frankfurt 1873. 396 Seiten, Oktav, mit Portrait (M. 7). M. 2.-.

180. S. Hedin, Minne af von Linné, Fader och Son. 2 Teile. Stockholm 1808. Oktav. Mit 2 Portraits u. 2 Tafeln.

181. O. E. A. Hjelt, Linné u. seine Bedeutung f. d. medizinische Wissenschaft. Leipzig, Engelmann, 1882. 100 Seiten, Oktav. (M. 2).

182. H. Malmstén, K. v. Linné. Berlin, Habel, 1879. 38 Seiten, Oktav.

183 D. H. Stöver, Leben d. Ritters C. v. Linné 2 Teile und Nachtrag. 432 u. 360 Seiten, Oktav. Mit Portrait. 184. Englische Uebersetzung von J. Trapp. London, White, 1794. 470 pages in Quarto with portrait and plate $(1 \not{E} 1 \mathrm{sh}$.$) . M. 4$.

-185. Zetterstedt, Minnesord öfv. Linné. Jönköping 1874. Oktav.

M. 2.-

Eine gute Analyse der' Schriften Linné's, soweit sie nicht posthum erschienen sind, giebt:

486- R. Pulteney, A general view of the writings of Linnaeus. London 1781. 425 pages in Octavo. M. $4 .-$

187. 2. edition, with corrections, considerable additions and memoirs of the author by W. G. Maton. London, -Taylor, 1805. 610 pages in Quarto with portrait and 4 plates. $(1 £ 12$ sh.). M. 8. - 188. French Translation: Revue générale des écrits de Linné. Traduit par L. A. Millian de Grandmaison. 2 volumes. Londres, Buisson, 1789. 390 et 400 pages in Octavo. M. 4.

Die Preise fast aller Linné'schen Werke, soweit sie Original-Ausgaben sind, befinden sich seit der Nomenclatur- Bewegung im. Steigen. Es ist interessant zu lesen, dass Brunet, den wir oben wiederholt citierten, 1862 schreiben konnte: Les ouvrages de Linné, quojque toujours fort estimés, sont beaucoup moins chers en ce moment qu'ils ne l'étaient il y a quarante ans, parce que les éditions multipliées qui en ont été faites les ont rendus plus communs (!), et que d'ailleurs, plusieurs d'entre enx sont déjà fort en arrière des progrès que l'histoire naturelle a faits dans ces derniers temps. - Auffällig ist aber, dass bei der jetzt so üblichen Publikation von GesamtAusgaben hervorragender Forscher noch nicht an die Veröffentlichung der gesammelten Werke Linné's gedacht worden ist. Die schwedische Akademie hat nur die Jugendschriften herausgegeben.

So gross wie bei keinem anderen Forscher und kennzeichnend für die dauernde Wertschätzung Linné's speziell für die Systematik der Naturwissenschaft sind die Gesellschaften und die Zeitschriften, die sich nach ihm nennen, so die Linnean Society in London und die in New South Wales, die Société Linnéenne in Bordeaux, Caen, Lyon, Paris, ferner die (eingegangenen) Zeitschriften Linnaea und Limnaea Entomologica, u. a.

Der unbedeutende Sohn des grossen Vaters: C. a Linné filiuis $(* 1741,+1783)$ hat publiciert:

189. Decas I (et II) Plantarum Rariorum Horti Upsaliensis. (quod prodiit). Upsala, Swederus, $1762-63$. 40 paginae in Folio cum 20 tabulis

Decas I ist 1767 in Leipzig von Crusius nachgedruckt.

Ausser obigem und 3 kleineren Dissertationen in 'Amoenitates' Band $\mathrm{X}$ ist von dem jüngeren Linné nichts erschienen. (Siehe auch Nr. 22).

Die „Decades“" steigen, da nicht häufig; im Preise.

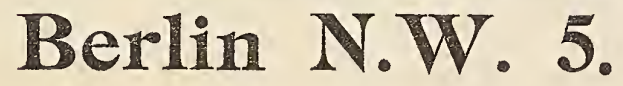

W. Junk. 


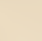




\section{W. Junk, $\begin{gathered}\text { Verlag fi. Naturmissensehaften } \\ \text { Scieutffie Publisher }\end{gathered}$ Berlin, N.W. 5.}

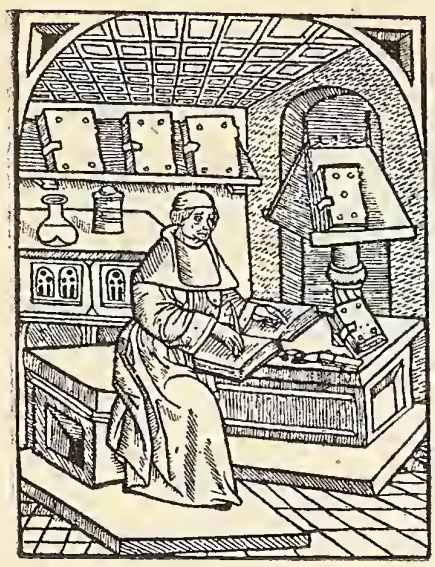

Soeben erschien:

Just published:

\section{Facsimile-Edition. Ed. W. Junk. No. II: B o ja $\mathbf{u s}$ Anatome Testudimis Europace.}

[Wilna 1819-21]. Folio. 190 paginae et 40 tabulae. M. 125 (=6 62 sh. = Doll. 29,50)

Das Buch von Bojanus, dessen Original-Ausgabe in kaum 80 Exemplaren horgestellt wurde, ist wohl das seltenste zoologische Werk. Im Laufe von 20 Jahren meiner ausschliesslich dem naturwissenschaftlichen Antiquariat gewidmeten Praxis ist mir nur ein einziges vollständiges Exemplar vorgekommen. Der Preis eines Originals ist bis 400 Mark gestiegen. Dabei ist das Werk von Bojanus für die vergleichende Anatomie heute noch unentbehrlich, da es das einzige ausführliche und (vorzüglich) illustrierte Buch über die Anatomie der Schildkröte ist.

Der Nachdruck ist mit den neuesten Mitteln der Reproduktionstechnik auf chemischem und photographischem Wege vorzïglich hergestellt und, um jedem Fxemplar gleiche Güte zu. wahren, nur in ganz geringer Zahl vervielfältigt worden.
The work by Bojanus, published in hardly 80 copies, is perhaps the rarest zoological book. During 20 years' occupation with scientific literature I have seen only one complete copy. The price of the work has risen to nearly twenty Pounds. - But the book is indispensable for the student of comparative anatomy being the only one treating at large and illustrating - in a superior manner - the Anatomy of the Turtle.

My chemical and photographic Reprint is made in an excellent way and - to ensure the same quality to every copy - only in a very limited edition which is nearly wholly subscribed.

1901 erschien:

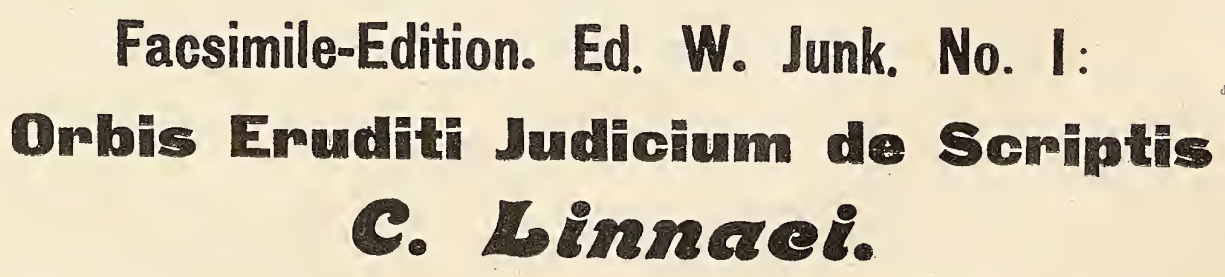

[Stockholm 1741]. Octavo. 16 pagiñae. M. 10 (=10 sh. = Doll. 2,40$)$.

Stöwer, Leben Linné's: „Die einzige besondere Apologie, die Iinné jemals für sich geschriebon. Aeusserst selten, selbst in Schweden. Weder Haller noch andere Literatoren scheinen sie gekannt zu haben, haben sie wenigstens nicht angeführt, und in Deutschland existiert sie vielleicht nicht in ein paar Exemplaren.

Prof. Nitsche-Tharandt ("Liter. Centralblatt"): Das kleine Büchlein ist der Facsimile-Druck einer von Linné selbst anonym herausgegebenen Schrift, die heute zu den grössten bibliographischen Seltenheiten gehört. Ausser diesem sind nur 3 weitere bekannt.

Prof. N. 0. Witt im "Prometheus" beginnt eine lange und sehr wohlwollende Beurteilung: Liebhaber bibliographischer Curiosa werden an dem hier angezeigten Facsimiledruck ihre Freude haben; derselbe bildet ein kleines Heftchen, welches mit Ausnahme des Umschlages eine absolut genaue, vermutlich auf photographischem Wege hergestellte Reproduktion eines der seltensten existierenden Druckwerke darstellt. Es sind iberhaupt in drei Exemplare des Originals bekaunt, und der Verleger des Neudruckes ist bestrebt, auch durch seine von ihm veranstaltete Ausgabe die Seltenheit des Werkes nicht allzu sehr herabzusetzen, indem er seine Facsimile-Ausgabe in nur 100 Exemplaren hat herstellen lassen. Abgeselien von dem bibliographischen Interesse, welches diese Erscheinung darbietet, ist sio auch in rein wissen. schaftlicher Hỉnsicht im hohen Grade bemerkenswert.

F. v. Zobeltitz (Zeitschr, f. Bücherfreunde): Höchst seltenes Werkchen . . Im ganzen dürften heute nicht mehr als 3 Exempl. bekannt sein ... Genau facsimilirt. 


28508 New York Botanical Garden Library

Z8508 J8 c.2
Junk, Wilhelm/Bibliographia Linnaeana. V gen

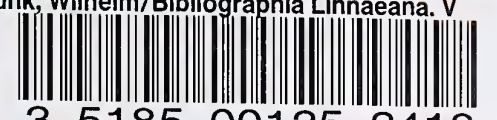

3 5185001252418 
